\title{
Successful suppression of magnetization precession after short field pulses
}

\author{
M. Bauer, R. Lopusnik, J. Fassbender ${ }^{a}$, and B. Hillebrands \\ Fachbereich Physik and Forschungs- und Entwicklungsschwerpunkt Materialwissenschaften, Universität Kaiserslautern, Erwin-Schrödinger- \\ Strasse 56, 67663 Kaiserslautern, Germany
}

\author{
H. Dötsch \\ Fachbereich Physik, Universität Osnabrück, 49069 Osnabrück, Germany
}

\begin{abstract}
For the next generation of high data rate magnetic recording above $1 \mathrm{Gbit} / \mathrm{s}$, a better understanding of the switching processes for both recording heads and media will be required. In order to maximize the switching speed for such devices, the magnetization precession after the magnetic field pulse termination needs to be suppressed to a maximum degree. It is demonstrated experimentally for ferrite films that the appropriate adjustment of the field pulse parameters and/or the static applied field may lead to a full suppression of the magnetization precession immediately upon termination of the field pulse. The suppression is explained by taking into account the actual direction of the magnetization with respect to the static field direction at the pulse termination.
\end{abstract}

\section{I) Introduction}

Short magnetic field pulses may be used in the near future to change the magnetization state in magnetic storage devices. It is of particular importance to avoid a large precession amplitude of the magnetization ("ringing") after a field pulse is used to switch the direction of magnetization. An appearance of ringing will increase the dead time required to make two consecutive switching events successful. Hence, investigations of the magnetization dynamics [1]-[6] have become technologically important.

It has been predicted by numerical simulations [7] and experimentally verified [8] that the magnetization ringing can be suppressed by tailoring the length of the applied field pulse. Moreover is has been demonstrated that the ringing in magnetization dynamics can also be suppressed by a variation of the time delay between the two steps in the step excitation [9].

In this paper it is shown that the ringing can also be suppressed by an appropriate adjustment of the static applied field, which mainly determines the precession frequency.

\section{II) Experimental}

In order to investigate the dynamic properties, a time resolved magneto-optical Kerr effect magnetometer [8] was used. Time resolution is realized through the ap- plication of magnetic field pulses to the sample, and subsequently by testing the magnetization state by means of the longitudinal magneto-optic Kerr effect using a time delayed, pulsed picosecond infrared diode laser. The laser pulses used here had a duration of 15 ps and an energy of $10 \mathrm{pJ} / \mathrm{pulse}$. The over-all time resolution of the setup was 100 ps.

Results are presented for a $\mathrm{Lu}_{2.04} \mathrm{Bi}_{0.96} \mathrm{Fe}_{5} \mathrm{O}_{12}$ (BIG) single crystal film grown by liquid phase epitaxy onto a (111)oriented gadolinium gallium garnet (GGG). The film has a thickness of $1.5 \mu \mathrm{m}$, a saturation magnetization $4 \pi \mathrm{M}_{\mathrm{s}}=1750 \mathrm{G}$, and a ferromagnetic resonance line width at half maximum of $0.9 \mathrm{Oe}$ at $8.1 \mathrm{GHz}$ [10]. The size of the sample was $1 \times 1 \mathrm{~mm}^{2}$. Iron garnet films are known to exhibit a very low magnetic damping constant [11], which allows to excite a pronounced ringing. Therefore, any method to suppress ringing can be rigorously tested.

\section{III) Ringing suppression by field pulse shaping}

The experiment has been performed in the following way (cf. inset of .1): The static magnetic field, $H_{\text {static }}=4 \mathrm{Oe}$, was applied in the film plane in order to create a preferred, single domain magnetization state with the direction of magnetization transverse to the plane of incidence. In-plane field pulses of about 0.6 Oe from a microstrip line were applied perpendicular to $H_{\text {static }}$. These field pulses cause a maximum tilt angle of the magnetization out of the static equilibrium direction of $9^{\circ}$. The rise and fall times of the magnetic field pulse are 2 ns. Never-

\footnotetext{
${ }^{\mathrm{a}}$ author to whom correspondence should be addressed. e-mail: fassbend@physik.uni-kl.de 
theless, magnetic field pulses $T_{\text {pulse }}<2$ ns can be achieved at the expense of the field pulse magnitude. The values of $T_{\text {pulse }}$ given below are specified at half maximum, while the delay time is measured from the launch of the field pulse. The time response of the magnetization during and after field pulse excitation was obtained for different field pulse durations, $T_{\text {pulse }}$.

The results are shown in Fig. 1. For the shortest magnetic field pulse used in these experiments, $T_{\text {pulse }}=1.4 \mathrm{~ns}$, a pronounced magnetization ringing upon field pulse termination is observed. By successive increase of $T_{\text {pulse }}$, the ringing amplitude decreases and vanishes for $T_{\text {pulse }}=3.2 \mathrm{~ns}$. A further increase in $T_{\text {pulse }}$ leads to the reappearance of pronounced ringing at $T_{\text {pulse }}=4.4 \mathrm{~ns}$. As a function of pulse duration, the ringing amplitude oscillates after the pulse termination with a suppression at $T_{\text {pulse }}=3.2 \mathrm{~ns}, 6.4 \mathrm{~ns}, 10.8 \mathrm{~ns}, 13.8 \mathrm{~ns}$, and $17.8 \mathrm{~ns}$ and pronounced ringing at intervening values of $T_{\text {pulse }}$. The suppression is most pronounced when comparing signals for $T_{\text {pulse }}=1.4 \mathrm{~ns}$ and $T_{\text {pulse }}=3.2 \mathrm{~ns}$. Note that although the ringing can be tailored after field pulse termination, during application of the field pulse the ringing behavior is not affected.

\section{IV) Ringing suppression by static field adjustment}

In a complementary experiment the effect of changes in $H_{\text {static }}$ on the ringing behavior was examined. In contrast to Sec. II, in-plane field pulses of 1.6 Oe were applied. The pulse duration was $18 \mathrm{~ns}$, and the rise/fall times were $0.4 \mathrm{~ns}$. The time response of the magnetization during and after field pulse excitation was obtained for different values of $H_{\text {static }}$.

These results are shown in Fig. 2. During the magnetic field pulse a pronounced ringing is always observed. An increase in $H_{\text {static }}$ causes three effects: i) The

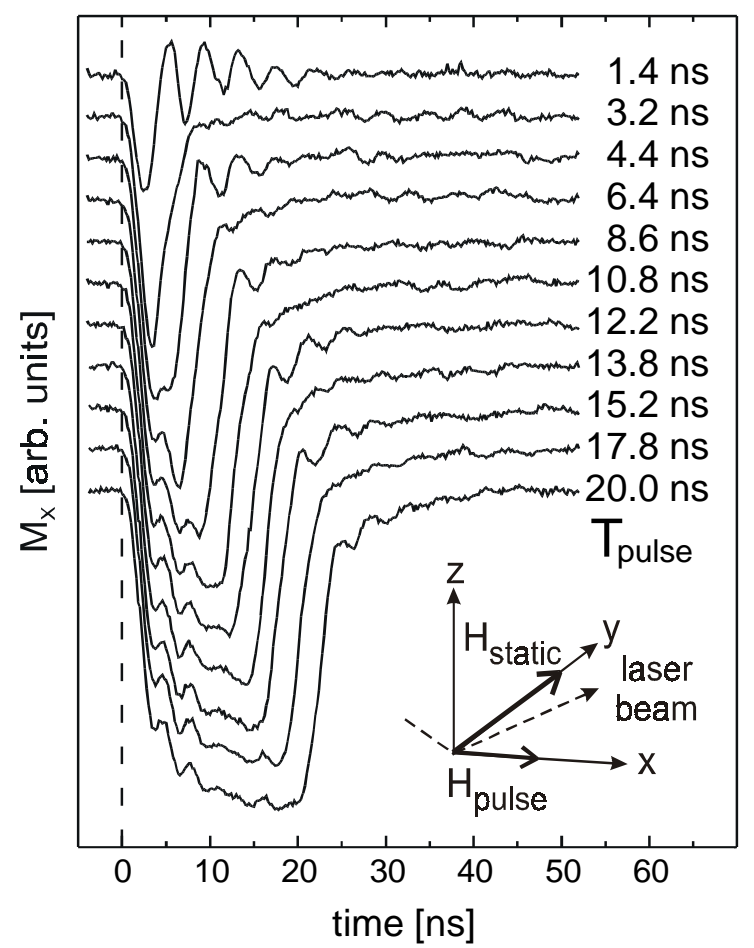

Figure 1: Time evolution of the $x$-component of the magnetization, $M_{x}$, measured at the center of the sample during and after field pulse excitation for different pulse durations, $T_{\text {pulse }}$, as indicated. The static magnetic field is $4 \mathrm{Oe}$, the magnetic field pulse $0.6 \mathrm{Oe}$. The beginning of the pulse launch is at time $t=0 \mathrm{~ns}$. Inset: Coordinate system and geometry of the experiment. precession frequency is increased. ii) Due to the constant pulse field magnitude the tilt angle between the magnetization and $H_{\text {static }}$ decreases. iii) The ringing amplitude decreases stronger than the tilt angle.

The ringing behavior after field pulse termination is mainly governed by the actual magnetization direction with respect to the static field direction. As a function of $H_{\text {static }}$, the ringing amplitude oscillates with a suppression at $H_{\text {static }}=24 \mathrm{Oe}, 28 \mathrm{Oe}$, and $34 \mathrm{Oe}$. For higher static fields the suppression is enhanced. A pronounced ringing is observed at values $H_{\text {static }}=21 \mathrm{Oe}, 26 \mathrm{Oe}, 32 \mathrm{Oe}$, and $38 \mathrm{Oe}$. In this case, the ratio of the ringing amplitude after and during field pulse application is enlarged with higher static fields. It is important to note that for two consecutive minima in the ringing amplitude the number, of precession periods during pulse application differs by one (see Fig. 2).

\section{V) Discussion}

In order to understand the experimental results, let us first discuss the simplified case of a rectangular pulse and a medium without shape anisotropy and damping. In this situation a periodic suppression of the dynamic magnetization ringing, which varies with either $T_{\text {pulse }}$ or $H_{\text {static }}$ can be explained by precession timing arguments. The precession may be considered in three steps. (i) Before the pulse, the magnetization is aligned parallel to $H_{\text {static }}$ and there is no precession. (ii) Once the pulse field is applied, the magnetization immediately begins precessional motion about the new effective field direction. (iii) In absence of damping, the precessing magnetization vector will periodi-

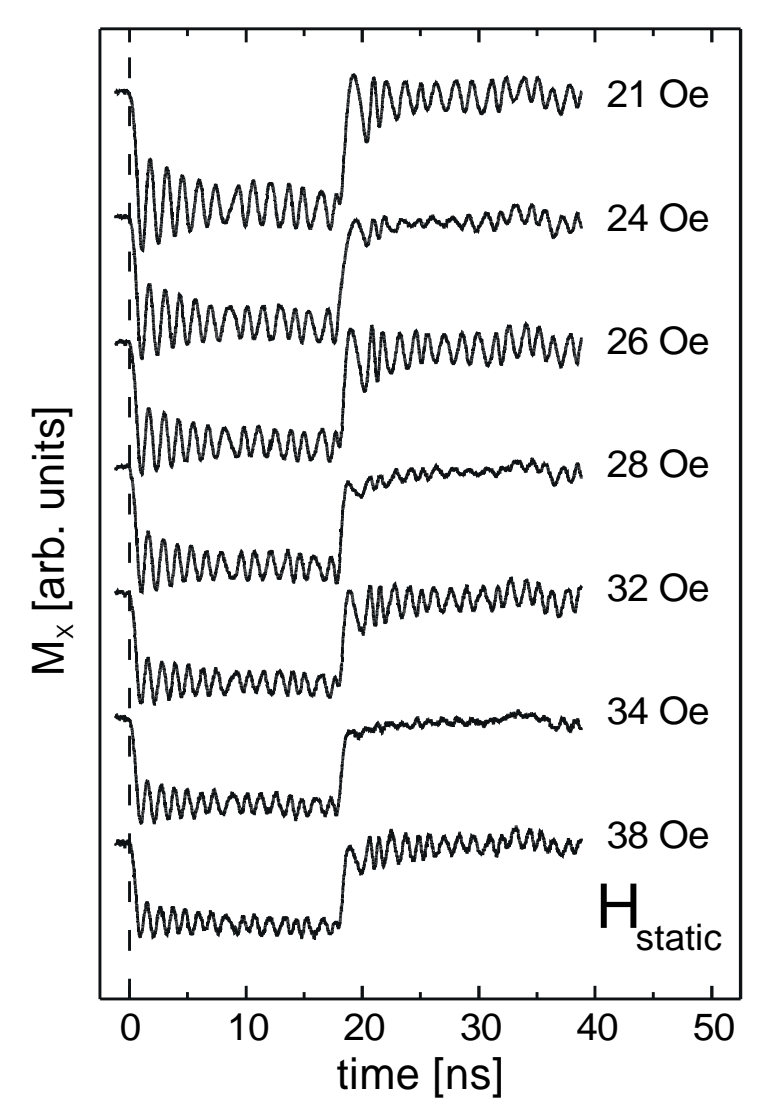

Figure 2: Time evolution of the $x$-component of the magnetization, $M_{x}$, measured at the center of the sample during and after field pulse excitation for different static magnetic field values, $H_{\text {static }}$, as indicated. The magnetic field pulse is $1.6 \mathrm{Oe}$ and the pulse duration is $18 \mathrm{~ns}$. The beginning of the pulse launch is at time $t=0 \mathrm{~ns}$. 
cally return to its initial position. If then the pulse field is immediately terminated the magnetization is already aligned with the static magnetic field and no ringing of the magnetization occurs. This scenario can be realized either by changing the pulse duration or the amplitude of the static applied field. Even so, for the inclusion of shape anisotropy, damping and a realistic pulse shape the basic concept remains the same although the actual magnetization motion is remarkably more complicated. In this case the successful suppression of magnetization ringing is possible as well, nevertheless it requires appropriate adjustment of the pulse field parameters and/or the static applied field.

\section{VI) Conclusion}

It has been shown experimentally that the magnetization precession after field pulse excitation can be suppressed by appropriate adjustment of the field pulse parameters and/or the static applied field. The effects are understood in terms of precession timing arguments. The main advantage of this technique over tailoring the magnetic damping mechanism is that the magnetic damping parameter has not to be increased in order to suppress the magnetization oscillations.

\section{Acknowledgement}

Support by Siemens AG, the German Ministry of Research and Technology (BMBF), and the European Union (TMR project Dynaspin) is gratefully acknowledged.

\section{References}

[1] W. K. Hiebert, A. Stankiewicz, M. R. Freeman, Phys. Rev. Lett. 79, 1134 (1997).

[2] M. R. Freeman, W. K. Hiebert, A. Stankiewicz, J. Appl. Phys. 83, 6217 (1998).

[3] R. H. Koch, J. G. Deak, D. W. Abraham, P. L. Touilloud, R. A. Altman, $\quad \mathrm{Yu} \quad \mathrm{Lu}, \quad \mathrm{W} . \quad$ J Gallagher, Phys. Rev. Lett. 81, 4512 (1998).

[4] T. J. Silva, C. S. Lee, T. M. Crawford, C. T. Rogers, J. Appl. Phys. 85, 7849 (1999).

[5] C. H. Back, J. Heidmann, J. McCord, IEEE Trans. Magn. 35, 637 (1999).

[6] T. M. Crawford, T. J. Silva, C. W. Teplin, C. T. Rogers, Appl. Phys. Lett. 74, 3386 (1999).

[7] M. Bauer, J. Fassbender, B. Hillebrands, and R. L. Stamps, Phys. Rev. B 61, 3410 (2000).

[8] M. Bauer, R. Lopusnik, J. Fassbender, B. Hillebrands, submitted to Appl. Phys. Lett.

[9] T. M. Crawford, P. Kabos, T. J. Silva, submitted to Appl. Phys. Lett.

[10]M. Bauer, C. Mathieu, S. O. Demokritov, B. Hillebrands, P. A. Kolodin, $\quad S$. Sure, H. Dötsch, V. Grimalsky, Yu. Rapoport, A. N. Slavin, Phys. Rev. B 56, R8483 (1997).

[11]Ferromagnetic Materials, ed. by E. P. Wohlfarth, Amsterdam, North-Holland (1980). 